\title{
Collaborative Human-Robot Manipulation of Highly Deformable Materials
}

\author{
Daniel Kruse, Richard J. Radke, and John T. Wen
}

\begin{abstract}
Robotic manipulation of highly deformable materials is inherently challenging due to the need to maintain tension and the high dimensionality of the state of the material. Past work in this area mostly focuses on generating a detailed model for the material and its interaction with the robot, then using the model to construct a motion plan. In this paper, we take a different approach by using only sensor feedback to dictate the robot motion.

We consider the collaborative manipulation of a deformable sheet between a person and a dual-armed robot (Baxter by Rethink Robotics). The robot is capable of contact sensing via joint torque sensors and is equipped with a head-mounted RGBd sensor. The robot senses contact force to maintain tension of the sheet, and in turn comply to the human motion. This is akin to handling a tablecloth with a partner but with one's eyes closed. To improve the response, we use the RGBd sensor to detect folds, and command the robot to move in an orthogonal direction to smooth them out. This is like handling cloth by looking at the cloth itself. Both controllers are able to follow human motion without excessive crimps in the sheet, but as expected, the hybrid controller combining force and vision outperforms the force controller alone in terms of tension force transient. The ability to quickly detect the state of the deformable material also enables more complex manipulation strategies in the future.
\end{abstract}

\section{INTRODUCTION}

The development of "human friendly robots" such as the Baxter from Rethink Robotics and the UR5/UR10 from Universal Robotics has stimulated research interest in problems that involve humans and robots in the same workspace. Human-robot collaboration includes diverse research, from anticipating a human's intention in a shared workspace, to generating human-friendly trajectories that are intuitive for a person to predict, to actually incorporating the human into the control loop. In this paper, we specifically address the problem of collaborative object manipulation, in which both a robot and a human are capable of affecting the state of the system. In particular, we address the problem of cloth manipulation, in which the human and robot grasp opposite ends of a piece of fabric, and the control objective for the robot is to keep the fabric taut while complying to the motions of the human operator.

Transporting large deformable materials is needed in certain manufacturing environments, for example those involving clothing or leather goods. Composites manufacturing also requires careful handling of resin-laden carbon fiber sheets to press over a mold layer by layer. In addition, several household tasks require deformable material handling such as

The authors are all with the Center for Automation Technologies and Systems and the Department of Electrical, Computer, and Systems Engineering at Rensselaer Polytechnic Institute, Troy, NY, 12180 $\{$ krused2, wenj\}@rpi.edu, rjradkedecse.rpi.edu laying a sheet on a bed or a tablecloth on a table. Currently, such tasks are all performed by human workers due to the need to maintain the appropriate amount of tension force (high enough to avoid sagging or wrinkles and low enough to avoid tearing).

Collaborative robot-robot or human-robot manipulation of a rigid body has long been studied by using various forms of hybrid position/force control. However, when the material is deformable, standard control strategies no longer apply since the force propagation in the material is far more complex. This paper addresses this more difficult problem with a combination of force-feedback control, which is most effective in ensuring compliance when the fabric is taut, and vision-feedback control, which is most effective in reestablishing tautness when the fabric is slack. In particular, we propose a novel approach for estimating the control directive for slack fabric based on the analysis of wrinkles in a color/depth image.

The control problem formulation is presented in Section II. Section III reviews related work in this area. Section IV presents the main contributions of the paper: the derivation of a hybrid control law using force- and vision-feedback for cloth manipulation and a model-free method for detecting the cloth state. We compare implementation results for both force-feedback and hybrid control laws in Section V.

\section{SYSTEM SETUP}

We define our task as consisting of two agents, one controllable (robot) and one uncontrollable (human), coupled to a highly deformable two-dimensional object akin to cloth or other fabrics. The controllable agent's goal is to minimize the amount of deformations or wrinkles imparted in the object from the uncontrollable agent, by either moving to comply with the uncontrollable agent's motion or by applying tension to maintain a taut state.

Throughout our development, we used the dual-armed Baxter robot, which has two seven degree-of-freedom arms, sensing capabilities for both the displacement and torque at each joint, and a suite of additional sensors. We augmented the Baxter robot by mounting an RGBd sensor (Microsoft Kinect) on its face screen to provide three-dimensional information about the scene.

Using these sensors, we gather information about the state of the system, as shown in Figure 1. Note that we do not have force sensors at the wrist, as is common, but instead we estimate the spatial force applied at the end effector from the measured joint torques:

$$
\tau=J(q)^{\top} f_{\mathrm{ext}},
$$




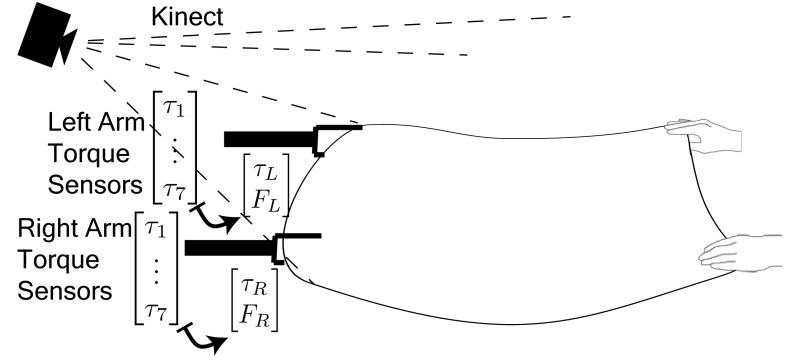

Fig. 1. The configuration for human-robot collaborative manipulation. The robot detects forces via torque sensors and maps them to end effector forces coming from the object. The Microsoft Kinect visually estimates the state of the deformable material.

where $f_{\text {ext }}=\left[\tau_{\text {ext }}, F_{\text {ext }}\right]^{\top}$ is the spatial force applied to the end effector, $J(q)$ is the Jacobian relating joint velocities to end effector twist, and $q$ is the vector of current joint angles.

We use this estimated force to follow the commanded forces of a human operator; however, since we are working with flexible materials, there is no guarantee that forces from the human will reflect through the material to affect the joint torques. In fact, when the material is in a "slack" state, the system is degenerate, and the person's motion will not reflect a detectable force through the cloth.

When in a slack state, visual feedback is necessary to interpret deformations in the cloth and determine the corresponding corrective movements to fully stretch the cloth back into a "taut" state. Here, we use an RGBd sensor, the Microsoft Kinect, for this task. We propose that instead of a full model approximation of the object state, we can instead detect deformations of the cloth from a direct sampling of points on its surface.

\section{RELATED WORK}

Substantial research has focused on forming representative models for flexible materials. Cloth and fabrics have received particular attention [2], [7], [9], [10], [14] due to their natural connections to everyday household life, but the manipulation of flexible materials extends to other fields, including industrial [3], [8] and biomedical [6] applications.

In all of these works, there was consensus that in order to manipulate deformable objects, at least two distinct grasping points are necessary, thus motivating our use of dual-armed robots. For a recent survey on dual-armed manipulation, we refer to [11].

Models for flexible materials typically consist of a mesh parameterized by a set of 3D vertices and edges; however, the high dimensionality of this representation can lead to algorithms with significant computational overhead. Minimal representations were proposed by Salzmann et al. [9], in which the cloth state is represented as a combination of learned deformation modes. However, the standard method for simulating deformable objects is with physics-based models, as in [3], [10].

Recent research has investigated the automated manipulation of flexible materials. Bodenhagen et al. [3] successfully performed peg-in-hole and laying down tasks with small elastic parts. Berenson [1] developed a heuristic-based control law in simulation that does not need an explicit model for deformable objects in tasks such as folding or wrapping around a rigid surface. The automated folding of laundry was presented in several unique papers [2], [5], [7]; however their manipulation processes used pre-planned motions based solely on geometric interpretation of the clothing.

Collaborative manipulation tasks between robots and humans have also received more interest with the recent introduction of commercial "safe" robots. Thobbi et al. [13] and Stuckler et al. [12] independently demonstrated the ability to jointly lift and move rigid objects of significant size relative to the robot; however, no work has been presented with deformable objects.

Though there is increasing interest in dual-arm manipulation of highly deformable materials, no purely sensor-based manipulation strategy has yet been proposed.

\section{CONTROL LAW}

We propose a hybrid controller comprised of two complementary pieces: one that uses force feedback and one that uses visual feedback. We designed velocity-input control schemes to meet the two primary objectives of 1) complying to human commands and 2) keeping the material free of significant deformations (i.e., taut).

\section{A. Force-Feedback Controller}

The Baxter robot is built using series elastic actuators that can sense the torque being applied by the motor via a spring connected directly to the actuator. Using (1), we can estimate the external force by solving for the inverse of this mapping. Baxter has two 7-dof redundant arms. If the arm is non-singular (i.e., the Jacobian matrix is full rank), we may estimate the applied force by

$$
f_{\mathrm{ext}}=\left(J^{\top}\right)^{\dagger} f_{d z}(\tau-g(q)),
$$

where $g(q)$ is the estimated gravity compensation torques for the joint configuration $q$, and $\left(J^{\top}\right)^{\dagger}$ is the Moore-Penrose pseudo-inverse of $J^{\top}$. The function $f_{d z}(\cdot)$ is a deadband, added to counter the effect of measurement noise.

With this estimation for the external forces applied to the system, we map these measured forces into velocities for the controller with a generalized damper control scheme. We apply a tension between the two grippers to keep the cloth edge taut. Using forward kinematics, we know the location of the two end effector locations $p_{O L}, p_{O R}$ in world space. Thus, the normal vector between the two is $h_{L R}=\left(p_{O R}-\right.$ $\left.p_{O L}\right) /\left(\left\|p_{O R}-p_{O L}\right\|\right)$.

We can reduce this tension control to a one-dimensional problem by projecting the measured forces onto this vector, as $\eta_{L}=-h_{L R}^{\top} F_{L}, \eta_{R}=h_{L R}^{\top} F_{R}$. The measured force then maps into desired velocities for each end effector based on error from a desired tension $\eta^{d}$ :

$$
v=-D^{-1}\left(\eta^{d}-\eta\right) h,
$$

where $D_{L R}$ is a damping constant, and $h$ is the normal vector onto which $F$ was projected. 
We define a nonlinear term $\zeta(\eta)$, as described in [4], for addressing contact constraints:

$$
\zeta(\eta)= \begin{cases}1 & \eta<0 \\ \frac{\eta_{1}-\left(1-\zeta_{f}\right) \eta}{\eta_{1}} & 0 \leq \eta<\eta_{1}, \\ \zeta_{f} & \eta \geq \eta_{1}\end{cases}
$$

where $\eta_{1}>0$ is a constant bias to ensure $\zeta(\eta)$ is a continuous function and $\zeta_{f} \in[0,1]$ is the desired scaling constant when $\eta$ exceeds $\eta_{1}$.

We use (4) to adjust (3) for each end effector as

$$
\begin{aligned}
& v_{L}=-\zeta\left(\eta^{d}-\eta_{L}\right) D_{L R}^{-1}\left(\eta^{d}-\eta_{L}\right) h_{L R}, \\
& v_{R}=\zeta\left(\eta^{d}-\eta_{R}\right) D_{L R}^{-1}\left(\eta^{d}-\eta_{R}\right) h_{L R} .
\end{aligned}
$$

Qualitatively, by introducing the $\zeta(\eta)$ term into the control law, the damping constant is increased when the tension force exceeds the desired value, causing a relatively slow decay back to equilibrium. This gives an implicit bias towards keeping the system in tension between the robot's grippers.

We extend this control into three dimensions by defining a coordinate system at each end effector as

$$
H_{L}=\left[\begin{array}{lll}
-h_{L R} & h_{\text {in }} & -h^{\perp}
\end{array}\right], H_{R}=\left[\begin{array}{lll}
h_{L R} & h_{\text {in }} & h^{\perp}
\end{array}\right],
$$

where $h_{\text {in }}, h^{\perp}$ are orthogonal vectors to $h_{L R}$ with $h_{\text {in }}$ pointing towards the body of the robot.

We can therefore extend (5) using (6) as

$$
v_{E}=-H_{E} D^{-1}\left(H_{E}^{\top} F_{E}-\eta^{d}\right), \quad E \in\{L, R\}
$$

where $D=\operatorname{diag}\left(\zeta D_{L R}, D_{\text {in }}, D^{\perp}\right)$ contains the damping coefficients for each tension direction, and $\eta_{d}$ is a vector of desired tension forces.

The joint velocities to drive the robot to a particular linear velocity are calculated using the Jacobian dampedleast-squares controller

$$
\dot{q}=J(q)^{\top}\left(J(q) J(q)^{\top}+\beta I\right)^{-1} v,
$$

where $J(q)$ is the $6 \times 7$ Jacobian matrix for a single arm, and $\beta$ is a small damping constant to avoid ill-conditioning in singularity configurations.

We include smooth joint-space force fields $\Psi_{q}(q)$ to avoid joint limits in the arms as a secondary control objective. We want this compensation to map into the null space of $J$, so as not to affect the end effector motion, so we adjust (8) with

$$
\dot{q}=J^{\top}\left(J J^{\top}+\beta I\right)^{-1}\left(v-J \Psi_{q}(q)\right)+\Psi_{q}(q) .
$$

In this way, we have robustness near joint limits using the arm redundancy to push into a safer pose without affecting our task-level control law.

\section{B. Vision-Feedback Controller}

We propose a fast method for estimating a corrective vector for slack cloth states based on observation of its wrinkles from the color-depth information provided by a Microsoft Kinect mounted on the face screen of the Baxter (Figure 2). We note that unlike many state-of-the-art techniques for cloth state estimation, no finite-element model for the cloth is required.

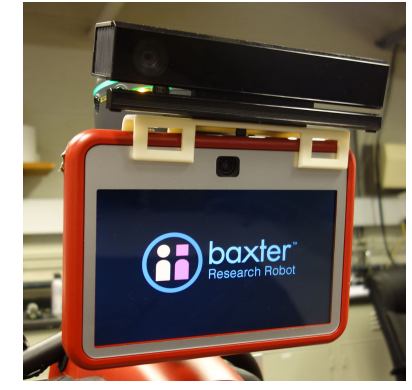

Fig. 2. Microsoft Kinect mounted on Baxter's facescreen.

Figure 3 illustrates the pipeline for processing the registered color-depth data from the Kinect. First, the cloth foreground is extracted from the background. We simply consider the depth image and remove edges between pixels that have significant depth discontinuities, followed by morphological open and fill operations on the binary image to remove spurious pixels (Figure 3b). Next, we color the foreground pixels using the registered RGB image, as shown in Figure $3 \mathrm{c}$, and remove any pixels dissimilar from the determined cloth color (Figure 3d).

We collect the remaining points into a set $\mathcal{P} \in \mathbb{R}^{3}$, where every point $p_{i} \in \mathcal{P}$ projects to a pixel $\left(u_{i}, v_{i}\right)$ in the RGB image $I$ based on the perspective projection model.

We next estimate the normal vector for each point $p_{i}$ based on its local neighborhood $\mathcal{N}\left(p_{i}\right) \in \mathcal{P}$. We remove any points in $\mathcal{P}$ without a fully populated neighborhood to prevent bias from missing points (shown in Figure $3 \mathrm{e}$ ). We collect second order information on $\mathcal{N}\left(p_{i}\right)$ into the $3 \times 3$ matrix

$$
M=\sum_{p_{j} \in \mathcal{N}\left(p_{i}\right)}\left(p_{j}-p_{i}\right)\left(p_{j}-p_{i}\right)^{\top} .
$$

The normal $h_{i}$, is the unit eigenvector associated with the smallest eigenvalue of $M$, with sign chosen to have a negative dot product with the optical axis of the Kinect. After processing each each point $p_{i} \in \mathcal{P}$, we get a set of corresponding normal vectors $h_{i} \in \mathcal{H}$.

We define the desired orientation for the cloth as a unit vector $h^{d}$, in this case the upward direction. Any normals that deviate from $h^{d}$ by a sufficient degree are considered as candidate directions for correction. Thus we define our candidate set as $\mathcal{H}^{c}=\left\{h_{i} \in \mathcal{H} \mid h_{i}^{\top} h^{d}<\delta\right\}$, where $\delta$ is a user-set threshold.

We estimate the corrective direction $\hat{h}^{c}$ as the mode of the candidate set $\mathcal{H}^{c}$ in spherical coordinates. As illustrated in Figure 4, this simple approach serves quite well to indicate the direction the cloth should be moved, in both tense and slack situations.

Finally, we define the vision-based task-space velocity control law for both arms as

$$
v_{C}=\frac{\left|\mathcal{H}^{c}\right|}{|\mathcal{H}|} v_{C \max } \hat{h}^{c},
$$

where $|\cdot|$ represents the cardinality of the set, and $v_{C \max }$ is the maximum velocity at which the vision-feedback controller 


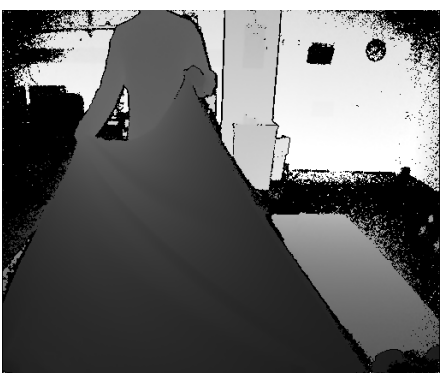

(a) Original Depth Image.

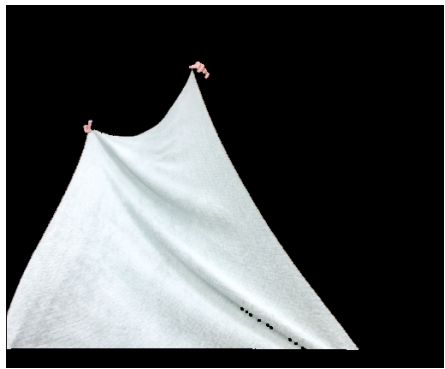

(d) Segment by color.

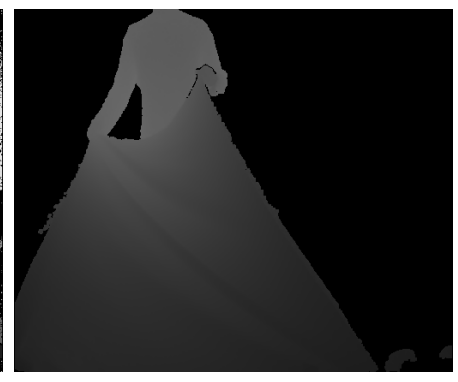

(b) Segment out foreground.

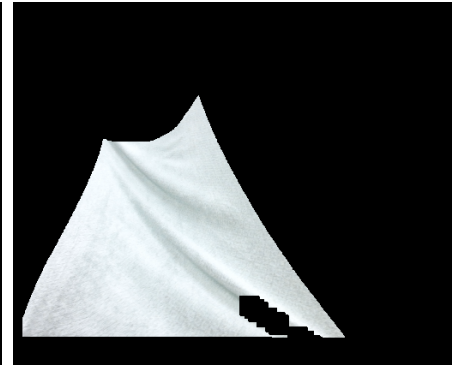

(e) Points with full neighborhoods.

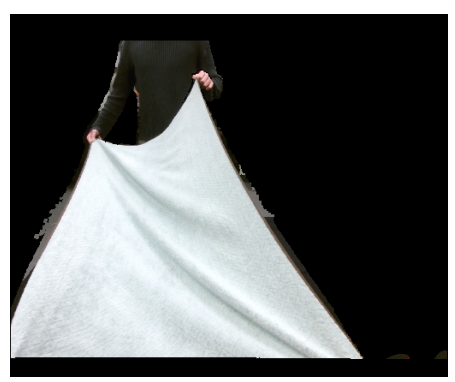

(c) Colored points.

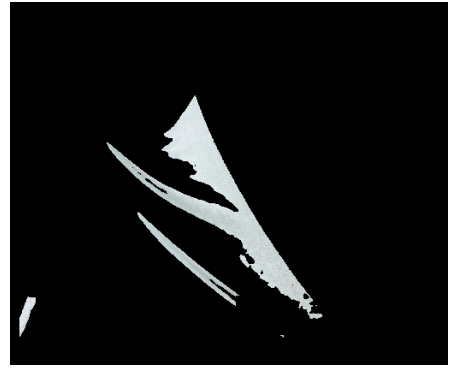

(f) Points that contribute to correction.

Fig. 3. The graphics pipeline for processing the RGBd data. The depth data in (a) is projected into 3D, and the background pixels are removed (b). We segment the colored points in (c) by the most prominent color to get (d). The points we actually compute surface estimations for are shown in (e), and (f) shows the points that contribute to estimating a corrective term.

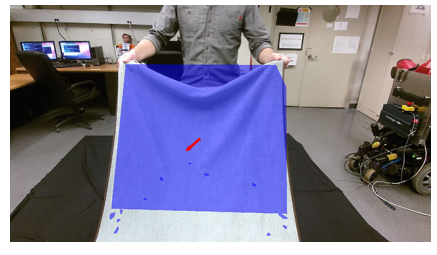

(a) $\mathrm{t}=1.895 \mathrm{~s}$

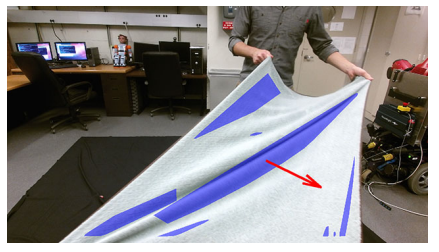

(b) $\mathrm{t}=6.7952 \mathrm{~s}$.

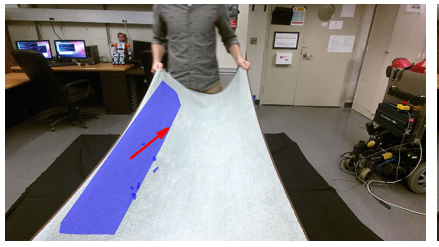

(c) $\mathrm{t}=12.0455 \mathrm{~s}$

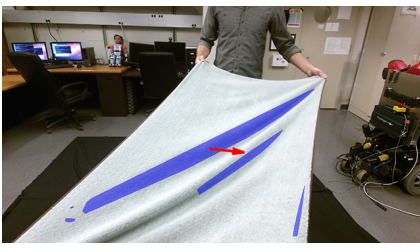

(d) $\mathrm{t}=23.246 \mathrm{~s}$.

Fig. 4. Selected frames from running the visual controller with a human operator. Visually estimated regions of deformation are labeled in blue, while the estimated correction vector to move the cloth (projected onto the image plane) is illustrated in red.

can operate. This controller then maps the task-space control law into joint velocities for both arms via (9).

While this control law is based on a heuristic, we argue that this process does drive the end effectors to position the grasped edge of the cloth towards the desired state. We can estimate the surface deformations in terms of curvature at each point, which fits nicely with our desired planar state for the cloth. If any point has a curvature $|\kappa|>0$, by definition this means that there is a change in the normal direction in that area. The principal directions for these curvatures closely relate to the internal forces applied at that position. We observed that the first principal direction is associated with compression, causing either buckling or drooping effects and the normals for a curve along this principal direction will point in the direction for the corrective term $\hat{h}^{c}$ for significant $\kappa$. By sampling these normals, we do not have to worry about the scale at which to estimate the curvature at each point, and can allows us to consider cases of both large deformation, as in Figure 4a, and local deformation, as in Figure 4b.

\section{Hybrid Force-Vision Controller}

The hybrid controller simply combines the task velocity terms from (7) and (11) into our full hybrid task-space control law

$$
v_{E}=-H_{E} D^{-1}\left(H_{E}^{\top} F_{E}-\eta^{d}\right)+\frac{\left|\mathcal{H}^{c}\right|}{|\mathcal{H}|} v_{C \max } \hat{h}^{c},
$$

where $E \in\{L, R\}$. The desired end effector velocities then map into joint velocities using (9).

The force-feedback control law can work on its own as a compliant controller, but there are inherent drawbacks to using force alone. As discussed earlier, since forces are only reflected during tension, there is a measured force of 0 when the cloth is slack. This means that force sensing alone cannot determine motion from the operator while in a slack state. Additionally, for maintaining tension between the two agents, there is a tradeoff between responsiveness and effort for the controller. From (3), while the fabric is slack, the maximum velocity that the end effector is commanded to move is $|v|=$ $\frac{1}{D} \eta^{d}$. Thus, for a desired $D$, the maximum velocity is tuned by $\eta^{d}$. This means that for small $\eta^{d}$, the robot can respond to 
lower forces when in tension, but will move respond slowly while slack. For high $\eta^{d}$, the robot will correct for slack more quickly, but will impart more tension force and require more effort from the person.

The vision-feedback controller also has drawbacks; however, they are complementary to those of the force-feedback controller. Notably, there is an inability to detect "outward" desired motion. When the human is pulling backwards on a taut sheet, the robot should comply and move "out" towards the person, but since we set the desired normal to the cloth $h^{d}$ as upward, the sensor would only observe a fully taut sheet without any surface deformations and (11) would be 0 . Thus, this controller is only able to supplement the forcefeedback controller, not stand on its own.

\section{RESULTS}

Each controller was implemented in MATLAB on separate computers running Windows with i7 processors. The scripts for each controller were communicated via the Robot Raconteur [15] middleware to the Robot Operating System (ROS) controller onboard the Baxter. Robot Raconteur is a middleware package similar to ROS, with support for Windows operating systems and the MATLAB language. The force-feedback controller sends commands at $\sim 50 \mathrm{~Hz}$, while the vision-feedback controller is significantly slower at $\sim 3 \mathrm{~Hz}$. In our experiments, we compared the performance between the force-feedback controller alone and the hybrid controller.

Even though this control scheme was designed for collaborative manipulation between a human and robot, we first present results using another Baxter running in open-loop as the operator. During experimentation, we set our $\eta^{d}=3 N$ for the desired tension between the grippers, as well as the tension between the two robots. Our test motions for the operator robot were generated to move both grippers in a sinusoidal path at $0.1 \mathrm{~Hz}$.

We tested the response from inward/outward motions; the positions and force results are shown in Figure 5. In Figures $5 \mathrm{a}$ and $5 \mathrm{~b}$ we see that there were only significant measured forces generated by pulling motions from the operator robot. Thus, as seen in Figure 5c, while the pure force controller tracks well when there is a measured force, in degenerate regions the end effectors can only move at a linear rate. The hybrid controller shown in Figure 5d has a much better tracking response in both directions, taking advantage of both controllers' strong points.

We also tested the response to side-to-side motions, with the results shown in Figure 6. We see from the force data in Figures $6 \mathrm{a}$ and $6 \mathrm{~b}$ that only one arm is receiving an external force at a time. This makes sense since, during sideto-side motions, the cloth undergoes a shear deformation. In this condition, the dominant force comes from pulling along the elongated diagonal. The position tracking, shown in Figure 6c, is poor during both control strategies, but shows improvement with the hybrid controller, as seen in Figure 6d. We attribute this muted improvement to the very little visual deformation of the sheet with respect to an upright pose.

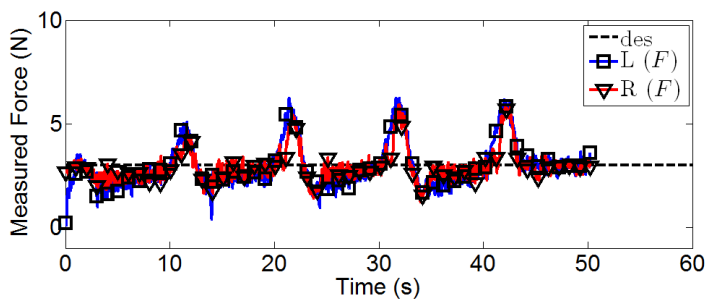

(a) Force-Feedback Force Response.

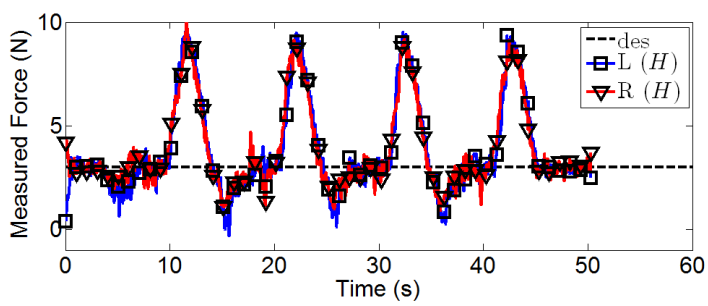

(b) Hybrid Force Response.

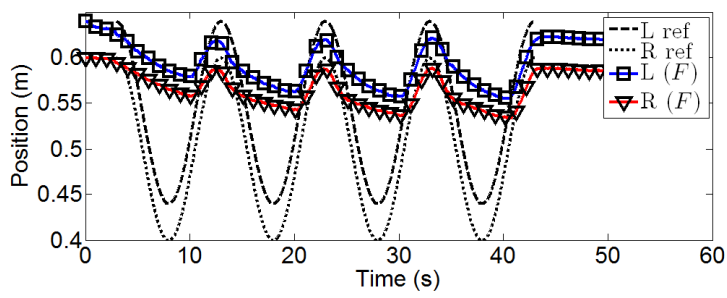

(c) Force-Feedback Position Response.

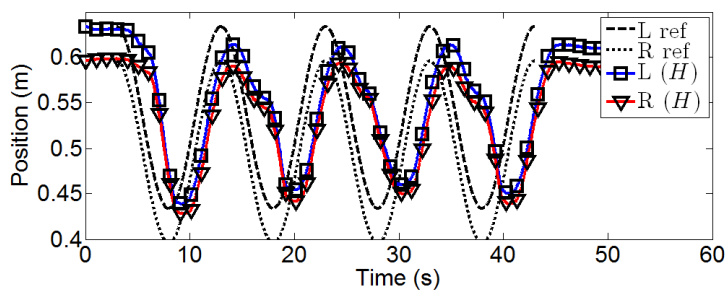

(d) Hybrid Position Response.

Fig. 5. The response for the compliant robot when the operator robot is making an inwards/outwards sinusoidal motion. Shown are both the left (L) and right (R) end effectors and the response from purely force control (F) and from the hybrid controller $(\mathrm{H})$.

Finally, we test the system using a human operator. Figure 4 illustrates several keyframes from this experiment. We observed similar behavior as with the controlled tests, and we refer the reader to the accompanying video for the full presentation of this test.

\section{DISCUSSION AND FUTURE WORK}

We presented a hybrid controller combining both force and vision data for a dual-armed robot to collaboratively manipulate cloth with a human. We showed that while a force-feedback controller is able to accomplish this task, the response is generally poor. The deficiencies of the force control come from degenerate states of the cloth when the material is slack; however, vision control performs very well in these regions, making the two controllers complement each other effectively. As a second contribution, we present a 


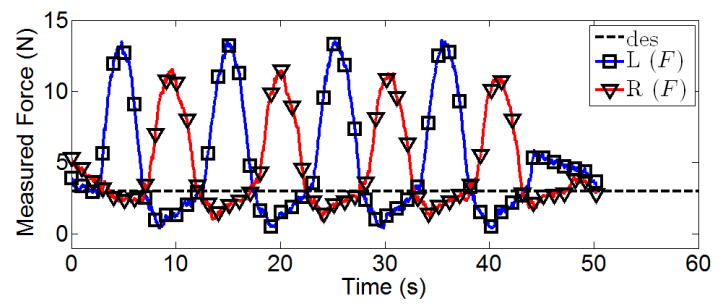

(a) Force-Feedback Force Response.

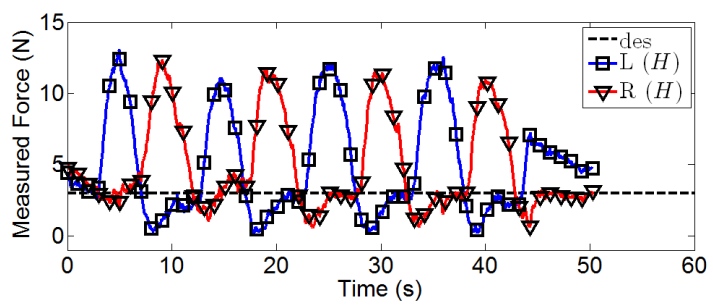

(b) Hybrid Force Response.

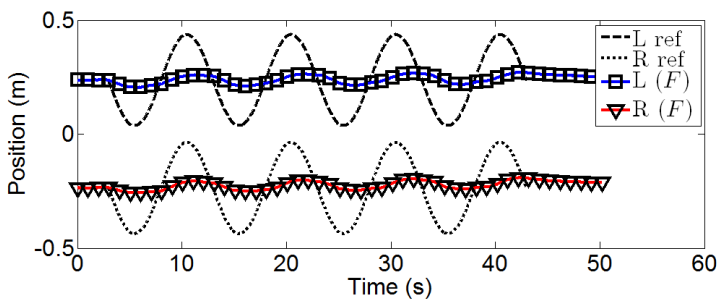

(c) Force-Feedback Position Response.

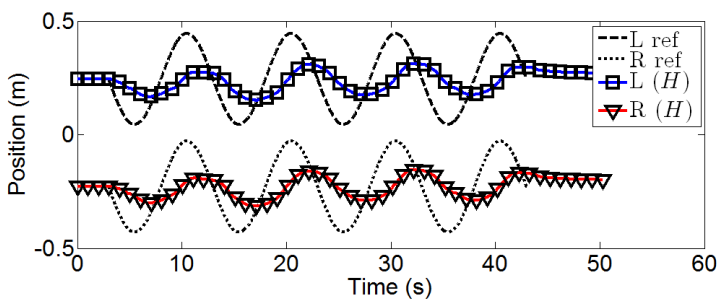

(d) Hybrid Position Response.

Fig. 6. The response for the compliant robot when the operator robot is making side-to-side sinusoidal motion. Shown are both the left (L) and right (R) end effectors and the response from purely force control (F) and from the hybrid controller $(\mathrm{H})$.

method to directly estimate local cloth states without a model for the cloth dimensions or its physical properties. We view this as a initial step towards more sophisticated future work in collaborative cloth manipulation.

The current vision-feedback controller estimates an appropriate corrective term for both arms as a vector perpendicular to the dominant deformation detected on the cloth surface. However, this is a heuristic control guideline and in the future, we are interested in a formal method to quantify the deviation from tautness of the cloth based on its surface geometry to drive an optimal correction step for each individual gripper. For example, our current visionfeedback controller would not correct the case when the robot's end effectors are too close, causing a symmetric droop with the cloth having two dominant normals pointing in opposite directions. In this case, the robot's hands would each need separate commands to spread apart. Future work will focus on modeling the relationship between end effector motion and the state change in local regions in the material. Additionally, our vision controller only detects a change in the system by observing the state of the cloth. We plan to extend this approach by predicting future states based on observations of the human motion.

\section{ACKNOWLEDGMENT}

The authors would like to thank John Wason for the development and sharing of Robot Raconteur, the distributed control middleware used in this research. This work is supported primarily by the Center for Automation Technologies and Systems (CATS) under a block grant from the New York State Empire State Development Division of Science, Technology and Innovation (NYSTAR).

\section{REFERENCES}

[1] D. Berenson. Manipulation of deformable objects without modeling and simulating deformation. In IEEE/RSJ International Conference on Intelligent Robots and Systems (IROS), Nov. 2013.

[2] C. Bersch, B. Pitzer, and S. Kammel. Bimanual robotic cloth manipulation for laundry folding. In IEEE/RSJ International Conference on Intelligent Robots and Systems (IROS), Sept. 2011.

[3] L. Bodenhagen, A. R. Fugl, A. Jordt, M. Willatzen, K. A. Andersen, M. M. Olsen, R. Koch, H. G. Petersen, and N. Kruger. An Adaptable Robot Vision System Performing Manipulation Actions With Flexible Objects. IEEE Transactions on Automation Science and Engineering, 11(3):749-765, July 2014.

[4] D. Kruse, J. Wen, and R. Radke. A sensor-based dual-arm tele-robotic system. IEEE Transactions on Automation Science and Engineering, 12(1):1-15, 2015

[5] J. Maitin-Shepard, M. Cusumano-Towner, J. Lei, and P. Abbeel. Cloth grasp point detection based on multiple-view geometric cues with application to robotic towel folding. In IEEE International Conference on Robotics and Automation (ICRA), May 2010.

[6] U. Meier, O. López, C. Monserrat, M. C. Juan, and M. Alcañiz. Realtime deformable models for surgery simulation: a survey. Computer methods and programs in biomedicine, 77(3):183-97, Mar. 2005.

[7] S. Miller, J. van den Berg, M. Fritz, T. Darrell, K. Goldberg, and P. Abbeel. A geometric approach to robotic laundry folding. International Journal of Robotics Research, 31(2):249-267, Dec. 2011.

[8] M. Saadat and P. Nan. Industrial applications of automatic manipulation of flexible materials. Industrial Robot: An International Journal, 29(5):434-442, 2002.

[9] M. Salzmann, J. Pilet, S. Ilic, and P. Fua. Surface deformation models for nonrigid 3D shape recovery. IEEE Transactions on Pattern Analysis and Machine Intelligence, 29(8):1481-7, Aug. 2007.

[10] J. Schulman, A. Lee, J. Ho, and P. Abbeel. Tracking deformable objects with point clouds. In IEEE International Conference on Robotics and Automation (ICRA), May 2013.

[11] C. Smith, Y. Karayiannidis, L. Nalpantidis, X. Gratal, P. Qi, D. V. Dimarogonas, and D. Kragic. Dual arm manipulation - A survey. Robotics and Autonomous Systems, 60(10):1340-1353, Oct. 2012.

[12] J. Stuckler and S. Behnke. Following human guidance to cooperatively carry a large object. In IEEE-RAS International Conference on Humanoid Robots, Oct. 2011.

[13] A. Thobbi, Y. Gu, and W. Sheng. Using human motion estimation for human-robot cooperative manipulation. In IEEE/RSJ International Conference on Intelligent Robots and Systems (IROS), Sept. 2011.

[14] P. C. Wang, S. Miller, M. Fritz, T. Darrell, and P. Abbeel. Perception for the manipulation of socks. In IEEE/RSJ International Conference on Intelligent Robots and Systems (IROS), Sept. 2011.

[15] J. Wason and J. Wen. Robot Raconteur: A communication architecture and library for robotic and automation systems. In IEEE International Conference on Robotics and Automation (ICRA), Aug. 2011. 